\title{
White Coat Hypertension in Aircrew: Diagnosis and Clinical Implications
}

\author{
Ahsan $\mathrm{MA}^{1}$, Rahman $\mathrm{MF}^{2}$, Munir $\mathrm{UR}^{3}, \mathrm{Ahmad} \mathrm{M}^{4}$, Sultana $\mathrm{SA}^{5}$ \\ DOI:https://doi.org/10.3329/jafmc.v16i2.55290
}

\begin{abstract}
Introduction: When there is a difference of patient's blood pressure (BP) which is measured in a physician's office and patient's home, it is said to have White Coat Hypertension (WCH). Prevalence of $\mathrm{WCH}$ is very much common among aircrew, because they need to face everyday preflight medical checkup.

Objective: To find out the incidence of WCH among the aircrew of Bangladesh Air Force (BAF) as well as to ascertain the long-term clinical implications of $\mathrm{WCH}$.

Materials and Methods: This cross-sectional observational study was conducted among 150 aircrew, reported to Central Medical Board (CMB), Bangladesh Air Force (BAF) for periodical medical evaluation, during the period January 2014 to December 2018. Study subjects were selected purposively with age range 25-50 years; those who did not have any history of hypertension or received any anti-hypertensive medication but were detected elevated $B P$ having clinical recording of $B P \geq 140 / 90 \mathrm{~mm}$ of $\mathrm{Hg}$. Ambulatory blood pressure monitoring (ABPM) was performed on them for 24 hours in order to distinguish between those who were truly hypertensive and those who were suffering from $\mathrm{WCH}$. To exclude secondary causes of hypertension as well as to assess for evidence of target organ damage (TOD), a thorough clinical and laboratory examination as well as regular follow-up were also performed.
\end{abstract}

Results: White Coat Hypertension was observed in $26.7 \%$ of the aircrew. There was no evidence of target organ involvement, such as left ventricular dysfunction, left ventricular hypertrophy, hypertensive retinopathy or renal involvement.

Conclusion: WCH is comparatively common among the aircrew. This highlights the importance of the requirement for regular follow up of all cases of WCH, over the normotensive subjects with a view to identify early organ involvement and therapeutic intervention are often initiated as and once needed.

Key-words: White-coat hypertension, Aircrew, BAF.

\section{Introduction}

Patients with white coat hypertension (WCH) blood pressure remains elevated when checked by a physician but remains normal in their daily life and at home ${ }^{1,2}$. WCH is defined by the European Society of Hypertension/Society of Cardiology guidelines 2013 as individuals with WCH are those who had office systolic/diastolic BP values of $140 / 90 \mathrm{mmHg}$ or higher at least three different occasions with ambulatory or home BP readings (24-hour ambulatory blood pressure $130 / 80 \mathrm{mmHg}$ or home blood pressure $135 / 85 \mathrm{mmHg})^{3,4}$. In contrast, ambulatory blood pressure measures show that 30 to $40 \%$ of individuals who are diagnosed with hypertension only by office BP measurement have normal out-of-office BP2. In certain cases, the most efficient way to diagnose and confirm whether individuals have chronic hypertension or white Coat Syndrome is by ambulatory monitoring ${ }^{5,6}$. Riva-Rocci identified WCH in 1896, describing it as a rise in blood pressure seen solely during a physician's visit. In 1983, researchers recorded intra-arterial blood pressure in hospitalized patients over a period of 24 hour ${ }^{4,7}$. When a physician appeared, the patient's blood pressure and pulse rate immediately increased. The patient's blood pressure rose 2-4 minutes after the start of the visit and stayed high throughout the duration of the physician's visit². Persons with $\mathrm{WCH}$ are with increased cardiovascular risk when office BP is $\geq 140 / 90 \mathrm{mmHg}$ and daytime ambulatory blood pressure monitoring (ABPM) is $<135 / 85 \mathrm{mmHg}$. BP is a continuous variable with no specific separation between normal and abnormal values. Similarly, there is a spectrum of definitions for day time ABPM that separate true sustained hypertension from WCH.

In this study, the incidence of $\mathrm{WCH}$ among the Bangladesh Air Force (BAF) aircrew were analyzed through periodic evaluation and assessed for any target organ involvement in these subjects with clinical significance of WCH and its implications with respect to the aviators.

\section{Materials and Methods}

This cross-sectional observational study using purposive sampling was carried out from Jan 2014 to Dec 2018 among the 150 aircrew who reported to Central Medical Board (CMB), BAF for periodical medical evaluation for flying duties and medical categorization. Inclusion criteria for this study were: asymptomatic aircrew, evidence of elevated BP recordings (office recording of systolic/ diastolic $B P \geq 140 / 90 \mathrm{~mm} \mathrm{Hg}$ ) minimum at three occasions, no prior or present history of intake of any antihypertensive medication or steroids. Persons having associated disease like diabetes or

1. Col Mohammad Ahmed Ahsan, MBBS, DAs Med (Pak), DAs Med (USA), MPH, Classified Specialist in Aerospace Medicine, Officer Commanding, Central Medical Board, Bangladesh Air Force (E-mail: ahsanahmed153@yahoo.com) 2. Maj Gen Md Fashiur Rahman, SPP, ndc, MBBS, MPH, LLB, FCGP, MBA, MSS, PhD Fellow, Ex-DGMS, Bangladesh Armed Forces 3. Col Umar Rashed Munir, MBBS, MPH, MBA (HRM), MPhil, PSA (HE), Assistant Director of Medical Services, 17 Infantry Division, Sylhet 4. Col Mushtaq Ahmad, MBBS, MCPS, DFM, FRSPH, Professor \& Head of Forensic Medicine, AFMC, Dhaka 5. Col Syeda Aleya Sultana, MBBS, D CARD (China), DAM, FCPS, FACC (USA), Classified Specialist in Medicine \& Cardiologist, CMH, Dhaka. 
ischemic heart disease or evidence of secondary hypertension were excluded from study. The protocol included a comprehensive history including family history, a detailed and thorough clinical evaluation to exclude any secondary causes of hypertension and to assess for target organ involvement or other associated diseases. All cases were then subjected to series of investigations including routine ABPM. All the aircrew were subjected to ultrasonography of the abdomen including the kidneys and adrenals, electrocardiogram (both resting and post exercise), chest X-ray (PA view) and 2D echocardiography in Combined Military Hospital, Dhaka.

All aircrew who formed the part of the study were then connected between $8.30 \mathrm{AM}$ and 12 noon to a 'Circadian BP Mate' BP monitoring system. The ABPM device was programmed to record the $\mathrm{BP}$ every 30 minutes during the day time and every 60 minutes during the night time $e^{8}$. The aircrews were instructed to keep their arm steady during measurements and to engage in normal activities between BP recordings. The mobile pressure level monitor was then disconnected after 24 hours on subsequent day. The BP recordings during the 24 hours' period, stored in the control module cum monitor, were then subsequently analyzed by a computer. For analysis $\geq 20$ reading during the day time and $\geq 07$ reading during the night were mandatory 8 . The ABPM was analyzed where systolic recordings found greater than $140 \mathrm{~mm} \mathrm{Hg}$ and diastolic recordings greater than $90 \mathrm{~mm} \mathrm{Hg}$, for the mean 24 hours' heartbeat and vital sign and additionally for the mean day time and evening time vital sign records. Person was labeling as WCH $^{0,10}$ with the criteria; (1) Office BP more $\geq 140 / 90 \mathrm{~mm} \mathrm{Hg}$ minimum at three occasions. (2) Aircrew with a 24 hours' average pressure level, on $A B P M \leq 135 / 85 \mathrm{~mm} \mathrm{Hg}$ and/or day time average onABPM $\leq 140 / 90 \mathrm{~mm} \mathrm{Hg}$. All recordings above these values were classified as primary high BP. Data were processed and analyzed using SPSS version 16. The tests of statistics used to analyze the data were descriptive statistics, Chi-square $\left(X_{2}\right)$ and unpaired t-test and the extent of significance was set at $p<0.05$.

\section{Results}

A total 150 aircrews of BAF, who were symptom free, were detected to be hypertensive on clinical evaluation, as per the inclusion criteria of the study. However, following analysis of the ABPM records of the 150 aircrew, $40(26.66 \%)$ were detected to have evidence of $\mathrm{WCH}$ and $20(13.33 \%)$ were finally diagnosed as primary hypertension. WCH subjects a higher percentage was observed in the younger age groups, 20(13.3\%) in those upto 35 years and 50(33.3\%) between the ages 36 to 45 years. In the older age groups, the incidence of WCH was less, while primary hypertension was more. Those between 46 and 50 years $15(10 \%)$ and above the age of 50 years $10(6.6 \%)$ had evidence of WCH (Table-I). All the cases of WCH were in the Grade I to II hypertension on clinical evaluation (diastolic BP in the range 90-109 mm of Hg). Out of 150 aircrews,20(13.33\%) had evidence of stage I hypertension and the remaining 20(13.33\%) aircrew had stage II hypertension. None of the cases of $\mathrm{WCH}$ had any evidence of target organ involvement. Furthermore, concentric LVH on 2D echocardiography was not evident in $\mathrm{WCH}$. The left ventricular ejection fractions were also well within the normal in all cases of $\mathrm{WCH}$. On the other hand, of the 20 cases detected to have primary hypertension, early hypertensive retinal changes were observed in 1 case and 1 of the cases had evidence of renal involvement. However, evidence of early concentric LVH was observed in 4 cases (Table-IV). All the150 aircrew evaluated by ABPM (40 with WCH \& 20 with primary hypertension) were ascertained to possess a traditional nocturnal dip of heart beat / pulse, high BP moves between $10 / 05 \mathrm{~mm} \mathrm{Hg}$ during the night/ sleep time recordings. None of the cases had evidence of nocturnal hypertension.

Table-l: Distribution of aircrew by age group $(n=150)$

\begin{tabular}{|c|c|c|c|c|}
\hline $\begin{array}{c}\text { Age } \\
\text { group } \\
\text { (years) }\end{array}$ & $\begin{array}{c}\text { All } \\
\text { subjects } \\
\mathrm{n}(\%) \\
\end{array}$ & $\begin{array}{l}\text { WCH } \\
\text { n (\%) }\end{array}$ & $\begin{array}{c}\text { Primary } \\
\text { hypertension } \\
\mathrm{n}(\%)\end{array}$ & $\begin{array}{c}p \\
\text { Value }\end{array}$ \\
\hline $25-30$ & $40(26.7)$ & $10(6.6)$ & $2(1.3)$ & \multirow{7}{*}{$<0.01$} \\
\hline $31-35$ & $35(23.3)$ & $10(6.6)$ & $2(1.3)$ & \\
\hline $36-40$ & $25(16.7)$ & $08(5.3)$ & $3(2.0)$ & \\
\hline $41-45$ & $25(16.7)$ & $06(4.0)$ & $4(2.7)$ & \\
\hline $46-50$ & $15(10.0)$ & $04(2.7)$ & $4(2.7)$ & \\
\hline$>50$ & $10(6.6)$ & $2(1.3)$ & $5(3.3)$ & \\
\hline Total & $150(100)$ & $40(26.7)$ & $20(13.3)$ & \\
\hline
\end{tabular}

Table-II: Distribution of aircrew with elevated BP (clinical records) and ABPM ( $n=150)$.

\begin{tabular}{|l|c|c|c|}
\hline \multicolumn{1}{|c|}{$\begin{array}{c}\text { Blood pressure } \\
\text { (Clinic recordings) }\end{array}$} & $\begin{array}{c}\text { Total number } \\
\text { ofcases } \\
\mathbf{n}(\%)\end{array}$ & $\begin{array}{c}\text { wCH } \\
\mathbf{n}(\%)\end{array}$ & $\begin{array}{c}\text { Primary } \\
\text { Hypertension } \\
\mathbf{n}(\%)\end{array}$ \\
\hline $\begin{array}{l}\text { Grade 1 Hypertension } \\
\text { (SBP/DBP: 140-159/90-99 mm Hg) }\end{array}$ & $100(66.7)$ & $20(13.3)$ & $11(7.3)$ \\
\hline $\begin{array}{l}\text { Grade 2 Hypertension } \\
\text { SBP/DBP: 160-179/100-109mm Hg }\end{array}$ & $50(33.3)$ & $20(13.3)$ & $9(6.0)$ \\
\hline $\begin{array}{l}\text { Grade 3 Hypertension } \\
\text { (SBP } \geq 1800 \text { o DBP } \geq 110 \mathrm{~mm} \mathrm{Hg})\end{array}$ & 0 & 0 & 0 \\
\hline Total & $\mathbf{1 5 0}(100)$ & $\mathbf{4 0 ( 2 6 . 7 )}$ & $\mathbf{2 0 ( 1 3 . 3 )}$ \\
\hline
\end{tabular}

*(SBP /DBP: Systolic blood pressure / Diastolic blood

Table-III: Target organ involvement in aircrew with hypertension $(\mathrm{n}=20)$

\begin{tabular}{|l|c|c|}
\hline Target organ involvement & Frequency & Percentage \\
\hline Hypertensive Retinopathy & 1 & 5 \\
\hline Renal involvement & 1 & 5 \\
\hline Concentric LVH & 4 & 20 \\
\hline
\end{tabular}

( $L V H=$ left ventricular hypertrophy)

\section{Dscussion}

WCH, a condition in which a patient's BP readings are higher when taken at the doctor's office compared to other settings, was originally attributed to the anxiety patients might experience during medical appointments. However, over the years, research has suggested the elevated readings might be a sign of underlying risk for future health problems. Microneurography, which monitors sympathetic nerve traffic in muscles and skin, has been used to 
measure and analyze $\mathrm{WCH}$. Muscle reflexes and emotional responses in skin nerves are responsible for the regulation of this function. This technique was used in a study on $\mathrm{WCH}$, which found that participants' skin nerves were activated and their muscle nerve traffic was inhibited when physicians took their blood pressure or were present during these measurements. Researchers indicate that this response is similar to a "defense reaction" that has been demonstrated in animal models when they react to emotional stressors. Therefore, emotional factors such as anxiety or stress may be responsible for this microneurographic response and the origin of $\mathrm{WCH}^{11,12}$. $\mathrm{WCH}$ is a common phenomenon noted in both hypertensive and normotensive subjects. Some authors have confidence that $\mathrm{WCH}$ is an intermediate stage between normotensive and hypertensive conditions ${ }^{13}$. A study demonstrated that patients with WCH condition had 2.5-fold higher chances to develop a sustained hypertension compared to normotensive subjects ${ }^{13}$. There is a link between persistent high blood pressure and the development of TOD and an increase in CV risk, thus it should no longer be regarded a harmless clinical condition.

Adults with WCH have a risk of developing LVH that is somewhere between normotensive and hypertensive ${ }^{14}$. Metabolic syndrome is linked with an increase in the risk for LVH in persons with WCH $(8 \%)$, whereas no cases were observed in control participants for that analysis ${ }^{15}$. In a study, $37 \%$ of subjects with WCH developed sustained hypertension over a mean follow-up time of 2.5 years with an accompanying rise in left ventricular mass ${ }^{16}$. To identify BP variation or $\mathrm{WCH}$, the most effective technique is to use an $\mathrm{ABPM}$. It was recently found in an extensive study by Banegas ${ }^{17}$ that $\mathrm{ABPM}$ is a stronger predictor of $\mathrm{CV}$ mortality and overall death than office measured blood pressure. Oscillometric monitoring of $\mathrm{BP}$ throughout 24 hours provides more accurate readings owing to diurnal and nocturnal variations ${ }^{18}$. ABPM also does not depend on the patient's handling, offers more exact $B P$ information about the individual's routine, reveals nocturnal hypertension, and analyzes the 24-hour efficacy of antihypertensive therapy ${ }^{19}$.

In this study involving a specific population, particularly skilled aircrew, showed higher incidence (26.66\%). This might be attributed to the very fact that these subjects are additional apprehensive as their career, flying prospects and finances are at stake, most likely creating them additional prone to $\mathrm{WCH}$ than the other population. It is recommended that both home blood pressure monitoring (HBPM) and automated BP monitoring (ABPM) be used to detect hypertension and white coat syndrome ${ }^{15}$. Individuals with $\mathrm{WCH}$ or hypertension should be closely monitored and identified in order to provide appropriate treatment measures due to the risk of deteriorating cardiovascular prognosis and the development of TOD. According to a new study headed by Penn Medicine experts and published in the Annals of Internal Medicine, persons with untreated $\mathrm{WCH}$ are twice as likely to die from heart disease as people with normal blood pressure ${ }^{20}$. Approximately one out of every five individuals may have $\mathrm{WCH}$, according to studies. A meta-analysis of 27 trials involving more than 60,000 individuals was done by the researchers in order to determine the cardiovascular hazards of $\mathrm{WCH}^{20}$. Patients with untreated $\mathrm{WCH}$ had a $36 \%$ higher risk of heart disease, a 33\% higher risk of mortality, and a 109 percent higher chance of dying from heart disease ${ }^{20}$.

\section{Conclusion}

There is an urgent need for expanded out-of-office blood pressure monitoring program across the country as it is crucial in the diagnosis and management of hypertension. WCH is a common prevalence among the aircrew who bear periodic medical checkup as a part of their analysis for flying fitness. According to different clinical research, "white coat" professionals may develop cardiovascular risks and lead to cardiac and vascular lesions. This underlines the importance of regular follow-up of all aircrew with WCH than the normotensive subjects. Aim of this is to observe early organ involvement in the variety of small proteinuria, retinopathy or cardiovascular involvement and to initiate therapeutic intervention as and once needed.

\section{References}

1. Celis H, Fagard RH. White-coat hypertension: A clinical review. Eur J Intern Med. 2004; 15(6):348-57.

2. Mancia G, Bombelli M, Seravalle $G$ et al. Diagnosis and management of patients with white-coat and masked hypertension. Nat Rev Cardiol. 2011; 8(12):686-93.

3. Mancia G, Fagard R, Narkiewicz K et al. 2013 ESH/ESC guidelines for the management of arterial hypertension: The Task Force for the Management of Arterial Hypertension of the European Society of Hypertension (ESH) and of the European Society of Cardiology (ESC) Eur Heart J. 2013; 34(28):2159-219.

4. Sipahioglu NT, Sipahioglu F. Closer looks at white-coat hypertension. World J Methodol. 2014; 4(3):144-50.

5. Franklin SS, Thijs L, Hansen TW et al. White-coathypertension: New insights from recent studies. Hypertension. 2013; 62(6):982-7.

6. Boggia J, Hansen T, Asayama K et al. White-coat Hypertension on Automated Blood Pressure Measurement Implicationsfor Clinical Practice. Eur J Cardiovasc Med. 2011; 1:17-21.

7. Mancia G, Bertinieri G, Grassi G et al. Effects of bloodpressure measurement by the doctor on patient's blood pressure and heartrate. Lancet. 1983; 2(8352):695-8.

8. Kikuya M, Hansen TW, Thijs L et al. International Database on Ambulatory blood pressure monitoring in relation to Cardiovascular Outcomes Investigators. Diagnostic thresholds for ambulatory blood pressure monitoring based on 10-year cardiovascular risk. Circulation. 2007; 115:2145-52.

9. Muntner $P$, Shimbo D, Carey RM et al. Measurement of Blood Pressure in Humans: A Scientific Statement from the American Heart Association. Hypertension. 2019; 73(5):e35-e66. 
10. O'Brien E, Parati G, Stergiou G et al. European Society of Hypertension Working Group on Blood Pressure Monitoring. European Society of Hypertension position paper on ambulatory blood pressure monitoring. J Hypertension. 2013; 31(9):1731-67.

11. Ralston SH, Penman ID, Strachan MWJ et al. Davidson's Principles and Practice of Medicine, 23rd Ed, 2018:508.

12. Grassi G, Turri C, Vailati S et al. Muscle and skin sympathetic nerve traffic during the "white-coat" effect. Circulation. 1999; 100(3):222-5.

13. Mancia $G$, Bombelli $M$, Facchetti $R$ et al. Long-term risk of sustained hypertension in white-coat or masked hypertension. Hypertension. 2009; 54(2):226-32.

14. Ihm SH, Youn HJ, Park CS et al. Target organ status in white-coat hypertensive's: Usefulness of serum procollagen type I propeptide in the respect of left ventricular diastolic dysfunction. Circ J. 2009; 73(1):100-5.

15. Mulè $G$, Nardi $E$, Cottone $S$ et al. Metabolic syndrome in subjects with white-coat hypertension: impact on left ventricular structure and function. J Hum Hypertension. 2007; 21(11):854-60.

16. Verdecchia P, Schillaci $G$, Borgioni $C$ et al. Identification of subjects with white-coat hypertension and persistently normal ambulatory blood pressure. Blood Press Monit. 1996; 1:217-22.

17. Banegas JR, Ruilope LM, de la Sierra A et al. Relationship between Clinic and Ambulatory Blood-Pressure Measurements and Mortality. N Engl J Med. 2018; 378(16):1509-20.

18. Sociedade Brasileira de Cardiologia (SBC), Sociedade Brasileira de Hipertensão (SBH); Sociedade Brasileira de Nefrologia (SBN). V Guidelines for ambulatory blood pressure monitoring (ABPM) and III Guidelines for home blood pressure monitoring (HBPM). Arq Bras Cardiol. 2011; 97(3 Suppl 3):1-24.

19. O'Brien E, Parati G, Stergiou G et al. European Society of Hypertension position paper on ambulatory blood pressure monitoring. J Hypertens. 2013; 31(9):1731-68.

20. Denker MG, Cohen DL, Townsend RR. People with untreated 'white coat hypertension' twice as likely to die from heart disease. Penn Medicine News, 10 June 2019. 